\title{
Manipulating the anisotropy of the Dirac-Cone in graphene by laser fields
}

\author{
Shahd Alfadhli ${ }^{1,2,3, a}$, Fedor V. Kusmartsev ${ }^{1,2,4, b}$, and Sergey E. Savel'ev ${ }^{2, c}$ \\ ${ }^{1}$ Microsystems and Terahertz Research Center, Chengdu, Sichuan 610200, P.R. China \\ ${ }^{2}$ Department of Physics, Loughborough University, Loughborough LE11 3TU, UK \\ ${ }^{3}$ Department of Physics, University of Tabuk, Tabuk 71491, Saudi Arabia \\ ${ }^{4}$ ITMO University, St. Petersburg 197101, Russia
}

Received 13 November 2018 / Received in final form 10 February 2019

Published online 15 April 2019

(C) The Author(s) 2019. This article is published with open access at Springerlink.com

\begin{abstract}
One of the most striking properties of graphene is the relativistic-like Dirac-Cone spectrum of charge carriers. By applying high-frequency laser fields, the system can be described with the use of similar spectrum which is based on a concept of electron quasi-energy. There in this spectrum a creation and annihilation of new Dirac points and cones as well as opening a gap may arise. This allows controlling electron motion without applying DC periodic fields which are effectively described by graphene superlattices. Here we demonstrate that coherent electromagnetic fields applied to graphene can generate new Dirac and Weyl points, induce Lifshitz quantum phase transition for slightly doped graphene and produce an anisotropy of the Dirac cones, which can be even infinite.
\end{abstract}

\section{Introduction}

Graphene has gained a huge interest since it has been discovered $[1,2]$. It is the basic structuring unit for building all graphitic materials [3]. Graphene has linear, gapless spectrum. The development of optoelectronic devices requires to open the gap. The issue of the energy gap opening and controlling in graphene has attracted a significant interest [4]. On the other hand, in order to maintain the carriers' high mobility in graphene, its energy spectrum should have linear Dirac form [5]. Hence, there is a demand for effective gap controlling i.e. opening, closing and new methods for manipulation of critical points such as minima and maxima of electronic zone in single layer graphene. The goal is to maintain the high carrier mobility. That will also allow a manipulation of Dirac points and cones such as suggested in references [6,7] as well as a creation of new 2D Weyl points and hyperbolic Dirac phase [8].

One of the most effective methods to control and to manipulate electron spectrum of graphene is to apply periodic electrostatic potentials (so-called graphene superlattices [9-13] and/or electro-magnetic fields. Recently, an analogy was found between electron spectrum in graphene superlattices and its modification in externally applied fields produced by laser [6]. The application of laser

\footnotetext{
${ }^{a}$ e-mail: Sh.a.f@outlook.com

b e-mail: F.Kusmartsev@lboro.ac.uk

${ }^{c}$ e-mail: S.Saveliev@lboro.ac.uk
}

fields to graphene creates a quasi-energy band gap structure (due to so-called a Stark effect, that is causing this gap opening). Moreover, the value of such gap can be easily controlled by changing electric field amplitude and frequency. Applying different frequencies or amplitudes of the laser field we are able to change the size and location of the gap in the spectrum in addition to forming new Dirac cones [6] which positions and form can be easily manipulated [7]. Nevertheless, the question remains if and how the laser-induced Dirac cones is distorted/squeezed near the laser-generated Dirac points. Typically in graphene, the constant energy map near Dirac points is expected to form circular curves. Here we derived and plot such curves from the dispersion relation which is the dependence of the quasi-energy on momentum $\vec{k}$. The natural analysis of our simulations leads us to the suggestions that besides Dirac points there arise Weyl points which appear always in pairs. Each pair of Weyl points corresponds to one Dirac point, where the first are topologically protected and the latter is only symmetrically protected. The creation and destruction of Dirac points therefore can be achieved by symmetry breaking, and, in particular, to inversion breaking. When this happen further tuning of electric field that breaks inversion will not remove Weyl points but only change their positions. The only case where Weyl points are removed is if we have two points at the same position with opposite chirality. Otherwise, the Dirac and 2D Weyl points have very similar behavior. They are both representations of similar Dirac and Weyl equations [7]. The Dirac points are 
formed in 3D topological insulators and they are topologically protected [7]. The Weyl point are formed mostly in semimetal and there are very robust in 3D space [14], e.g. different classes of topological Weyl semimetals have been described [8]. One of these types is the 3D Dirac semimetal is distinguished by its Dirac cone having a fourfold degeneracy. It emerges from twofold degeneracy of the time symmetry reversal ( $\mathrm{T}$ invariance) plus twofold degeneracy of the spatial inversion symmetry (P invariance). Another topological metal type is Weyl semimetals, which appears in the breaking of either symmetries ( $\mathrm{T}$ or $\mathrm{P}$ ). Thus, this type has a twofold degeneracy cones forming two Weyl points, which always appears in pairs. The pair hold two different topological charges +1 and -1 , caused by the chirality of the Weyl fermions. Therefore, Weyl points form some kind of "magnetic" monopoles and anti-monopoles in momentum space.

Below we describe a few different situations where 2D Weyl points is originating from a deformation of the quasienergy spectrum induced by external electromagnetic field. In particular that field creates the oval like singularity in the quasi-energy spectrum, and forms an anisotropic Dirac cones. Moreover we consider in details a similar diverse modification of the electron energy spectrum of graphene superlattices. We focus on the cases when both periodic static electric/magnetic field and laser field are applied to a single graphene layer. We show that the creation of the cones can be controlled by either periodic static magnetic or electric field and their shape depend on the amplitudes and frequencies of laser field. In particular at some critical field value the Dirac cone anisotropy changes drastically from fully symmetric, circular one to infinitely asymmetric, the completely squeezed one. That changing quasi-energy spectrum anisotropy may induce interesting anisotropic graphene properties, e.g. the Klein tunnelling induced by the laser light irradiation. We believe that such an effect can be easily experimentally verified.

\section{Model of graphene placed in linear resonator}

The dynamics of charge carrier in graphene placed in linear resonator are described by 2D Dirac equation, which can take the following form considering the natural units $e=\hbar=v_{F}=1[6]:$

$$
\left\{\vec{\sigma} \cdot\left[\vec{p}-\vec{A}(t)-\vec{A}^{H}(x)\right]+U(x)\right\} \psi_{A, B}=\frac{i \partial \psi_{A, B}}{\partial t}
$$

where $t$ is the time, $\vec{\sigma}$ is pauli matrices and $\psi_{A, B}=$ $\left(\begin{array}{l}\psi_{A} \\ \psi_{B}\end{array}\right)$ is the wavefunction describing charge carriers on the two triangular sublattices $A$ and $B$ of graphene honeycomb lattice. The resonator is characterised by the following distributions of the electromagnetic fields inside the resonating cavity forming a standing wave. The first component of this wave is the time periodic electric field, which can be created by laser irradiation of the resonator. It is represented by the vector potential $\vec{A}(t)$ :

$$
\vec{A}(t)_{x}=A_{0} \cos (\theta) \cos (\omega t) ; \vec{A}(t)_{y}=A_{0} \sin (\theta) \cos (\omega t)
$$

where $A_{0}$ is the amplitude, the frequency is $\omega=\frac{2 \pi}{T}$, and $\theta$ is the angle between the dynamic laser and static magnetic fields. The magnetic field inside the resonator is described by the vector potential: $\vec{A}^{H}(x)=\left(\vec{A}^{H}(x), \vec{A}_{y}^{H}(x)\right)$, where this can be a spatially periodic magnetic field, which is oriented perpendicular to the graphene layer as

$$
\vec{A}^{H}(x)_{y}=A_{0}^{H} \cos \left(\mu_{H} x\right)
$$

with amplitude of $A_{0}^{H}$ and frequency $\mu_{H}$. There inside the resonator exists also a static periodic electric field oriented in plane of the graphene. It is described by the potential:

$$
U(x)=U_{0} \cos (\mu x)
$$

with $U_{0}$ is its amplitude and $\mu$ is its period. Such configuration of the electromagnetic field may be achieved by several means. Due the two-dimensional geometry, graphene is perfectly fit for enclosure within different micro-cavities. Any photonic structures or interferometers that confines optical fields between two highly reflecting mirrors with a spacing of many wavelengths of light may form the desirable structure which induce the described field configuration. Two counter propagating laser beams is another alternative. So far it was popular to place graphene in such cavities, where graphene plane was parallel to the mirrors plane [15] or the laser beams was directed perpendicular to the graphene layer $[16,17]$. The main goal of these papers to find means of controlling the otherwise featureless optical absorption. The usage of waveguides in another means to make such field configuration [18]. Deposition of metal grading helps to create periodic electric field [19]. In all these systems the light-matter interaction is strongly enhanced, and they open up the opportunity for investigating fundamental changes in graphene it-self. In particular, here we show that there in slightly-doped graphene may arise a series of Lifshitz quantum phase transitions induced by applied electro-magnetic fields and strong light absorption observed in these systems [14-19] may be related to the light transformation of the graphene energy spectrum.

Floquet theory plays an essential role in analysing dynamical systems, where periodic systems can be transformed to linear forms. The Hamiltonian is periodic in time, with period of $T$, and in space with the same spatial periodicity as the crystal. Floquet theory illustrates that time periodic potentials transfers the electron's energy into a quasi-energy confined within its Brillouin zone and gives a solution of the form:

$$
\Psi(x, t)=e^{-i \varepsilon t} \Phi(x, t) .
$$




$\left[\begin{array}{cccccccc}\frac{\omega}{2} & k+C f & C_{n} U_{0} & i C_{m} A_{0}^{H} & 0 & -\left(\frac{A_{0}}{2}\right) e^{-i \theta} & 0 & 0 \\ k^{*}+C f & \frac{\omega}{2} & -i C_{m} A_{0}^{H} & C_{n} U_{0} & -\left(\frac{A_{0}}{2}\right) e^{i \theta} & 0 & 0 & 0 \\ C_{n} U_{0} & i C_{m} A_{0}^{H} & \frac{\omega}{2} & k+C f & 0 & 0 & 0 & -\left(\frac{A_{0}}{2}\right) e^{-i \theta} \\ -i C_{m} A_{0}^{H} & C_{n} U_{0} & k^{*}+C f & \frac{\omega}{2} & 0 & 0 & -\left(\frac{A_{0}}{2}\right) e^{i \theta} & 0 \\ 0 & -\left(\frac{A_{0}}{2}\right) e^{-i \theta} & 0 & 0 & \frac{-\omega}{2} & k+C f & C_{n} U_{0} & i C_{m} A_{0}^{H} \\ -\left(\frac{A_{0}}{2}\right) e^{i \theta} & 0 & 0 & 0 & k^{*}+C f & \frac{-\omega}{2} & -i C_{m} A_{0}^{H} & C_{n} U_{0} \\ 0 & 0 & 0 & -\left(\frac{A_{0}}{2}\right) e^{-i \theta} & C_{n} U_{0} & i C_{m} A_{0}^{H} & \frac{-\omega}{2} & k+C f \\ 0 & 0 & -\left(\frac{A_{0}}{2}\right) e^{i \theta} & 0 & -i C_{m} A_{0}^{H} & C_{n} U_{0} & k^{*}+C f & \frac{-\omega}{2}\end{array}\right]$

Given that $\Phi(x, t)$ has the same time periodicity as the Hamiltonian, and $\varepsilon$ is the quasienergy or Floquet energy. Within the resonance approximation [6], the wave function can be expanded as:

$$
\begin{aligned}
\psi_{A, B}= & e^{-i \varepsilon t+i k_{x} x+i k_{y} y}\left[\psi_{A, B}^{++} e^{\frac{i \omega t}{2}+\frac{i \mu x}{2}}+\psi_{A, B}^{+-} e^{\frac{i \omega t}{2}-\frac{i \mu x}{2}}\right. \\
& \left.+\psi_{A, B}^{-+} e^{\frac{-i \omega t}{2}+\frac{i \mu x}{2}}+\psi_{A, B}^{--} e^{\frac{-i \omega t}{2}-\frac{i \mu x}{2}}\right]
\end{aligned}
$$

where $\omega$ is the temporal frequency of the laser field, and $\mu$ is the spatial frequency of the spatially-periodic electric/magnetic field. Sine the Hamiltonian is timedependent, then the $\Phi(x, t)$ states should represent the stationary energy eigenstates, thus $\varepsilon=E$ (the quasienergy eigenvalue). In this paper, we applied a linearly polarized laser field to a single layer graphene [6,20]. The 2D Dirac Hamiltonian provides a differential equation, which describes the graphene subjected to the applied potentials. Substituting the wavefunction in a form of suggested by Floquet theorem [21], and restricting ourselves with several first harmonic of the corresponding Fourier expansion we obtain the matrix, which plays now a role of Hamiltonian:

\section{See equation (7) above}

where $k=k_{x}-i k_{y}$, hence, $k^{*}=k_{x}+i k_{y}, C, C_{n} \& C_{m}$ are constants that can be determined by the initial conditions, $A_{0}^{H}$ is the amplitude of the magnetic potential, $U_{0}$ is the amplitude of the electric potential, $f=\mu$ or $k_{0}$ is the frequency of the applied potential, $\omega$ and $A_{0}$ are the amplitude and the frequency of electromagnetic field from the lases. In the case of circularly polarised laser $A_{0}$ will be multiplied in the matrix by the coefficient $\left(1 \pm i e^{i \varphi}\right)$, and $\theta$ is the angle between an orientation of the static and dynamic fields. The zeros of the determinant for this matrix allow to determine the quasi-energy spectrum $\varepsilon\left(k_{x}, k_{y}\right)$ at any fixed applied field parameters.

\section{Graphene superlattices in static magnetic field formed inside resonator}

We consider an applied static magnetic field described by the vector potential $\vec{A}^{H}(x)$. It is oriented perpendicularly to the graphene sheet. This corresponds to the situation when graphene has been put in linear resonator. Then we also apply the electromagnetic field $d \vec{A}(t) / d t$ with electric component in plane assuming $U_{0}=0$. This fields are forming a standing wave in the resonator. Solving the 2D Dirac equation for Floquet states we obtain the quasi-energy spectrum near $\varepsilon=0$ (Fig. 1a). The spectrum consists of two linear nearly isotropic cones, where each holds two Dirac points (Fig. 2). It has four zero energy points in total. In the plane $k_{y}=0$ we see that each pair of Dirac points is created by an intersection of two parabolic curves, while in the planes $k_{x}=0.45$, and $k_{x}=0.55$ the spectrum is linear (see Figs. 2b and 2c). We found that the spectrum is very sensitive to the value of the frequency and amplitude of electromagnetic field applied.

At different value of frequencies $\omega$ of the resonator the spectrum has a different shape and the number of Dirac points, e.g. in agreement with our preliminary studies [21]. Overall, the spectrum can be described by a cross-section of the cones and a plane corresponding to a constant energy. We choose the value of quasi-energy close to zero. The results of the cross-sections are loops in momentum space and may be viewed as "Fermi surface" assuming the doping level lifts the Fermi energy from a Dirac point to this quasi-energy value. Such "Fermi surface" has always the shape of a few loops or their pairs reminding in general the shape of Cassini ovals (see Fig. 3). At small value of $\omega$ it is starting with two loops - two Cassini ovals, and then, with increasing $\omega$, it is evolving into three and then into four loops. Such transformation of Fermi surface is usually called as Lifshitz quantum phase transitions [22]. Each Fermi loop here has two fixed foci points $( \pm A, 0)$, and the product of the distance from any point on the spectrum to the foci's is fixed by the value $B^{2}$. The curve of each such Fermi loop has an equation of the form:

$$
\left(\left(k_{x}-A\right)^{2}+k_{y}^{2}\right)\left(\left(k_{x}+A\right)^{2}+k_{y}^{2}\right)=B^{2} .
$$

We can identify here three different regimes (phases), originated for different frequency range: (1) small $(\omega<0.5)$, (2) intermediate $(0.5<\omega<1.5)$, (3) for the specific particular frequency value, $\omega=1$, and (4) for large $\omega>1.5$. Here $\omega$ is measured in units of $\pi / T$. The transformation between these phases are due to the quantum Lifshitz phase transitions, which are similar to those described in reference [22]. 


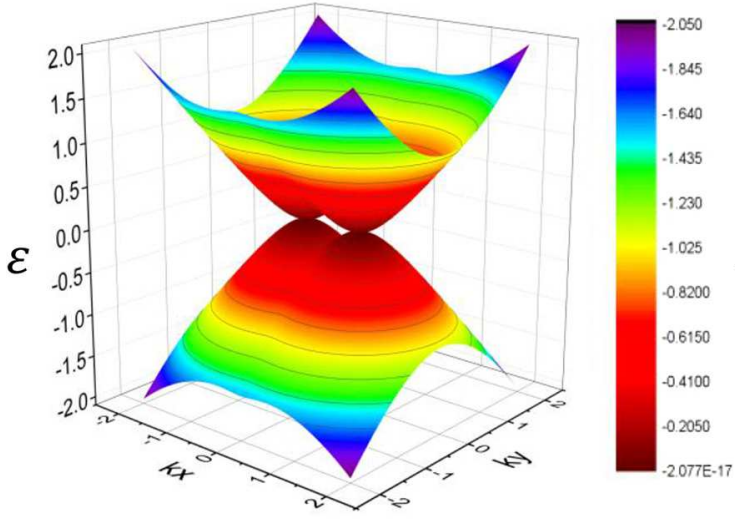

(a)

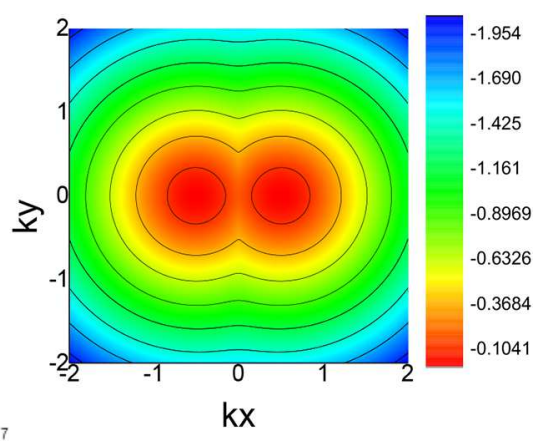

(b)

Fig. 1. The quasienergy spectrum of graphene in a linearly polarised laser and a static magnetic fields calculated at the following values of the parameters: $\omega=0.1, \mu_{H}=1$, measured in the units of $\pi / T$ and $A_{0}^{H}=1$ (a) 3D plot of the spectrum showing two points at which upper and lower surfaces are touching each other, where $\varepsilon\left(k_{x}, k_{y}\right)=0$. (b) The contour plot for this dependence of the energy on momentum, when the energy is positive, see (a).

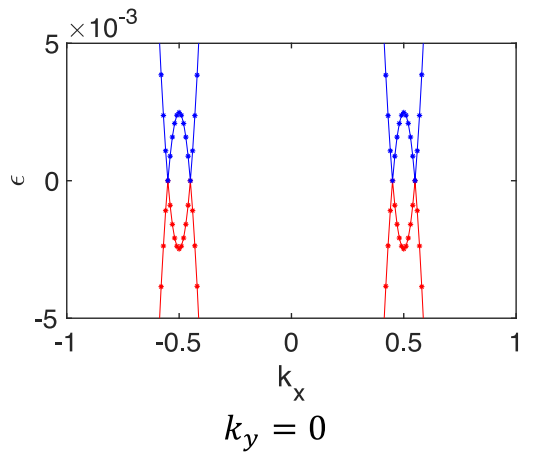

(a)

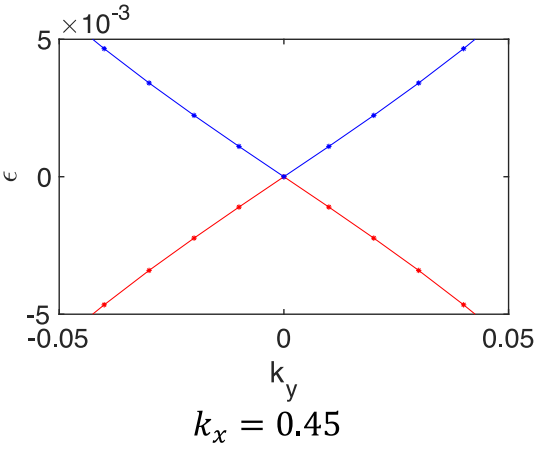

(b)

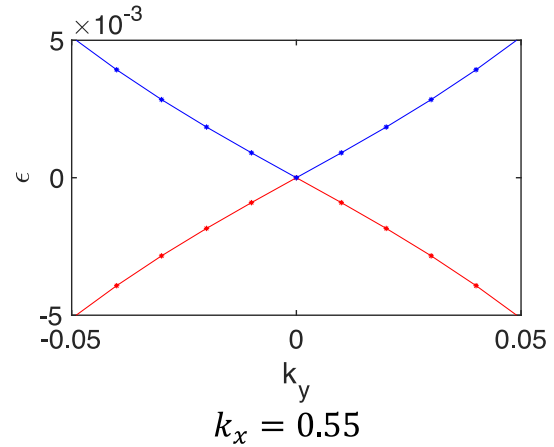

(c)

Fig. 2. The cross-sections of the linear dispersion relation of electrons in graphene placed in linear resonator taken in the planes (a) $k_{y}=0$; (b) $k_{x}=0.45$ and (c) $k_{x}=0.55$. Such complex shape of the spectrum is also arising at $\mathrm{K}^{\prime}$ point.

At small frequencies $(\omega<0.5)$, two Dirac-like cones have fixed foci points $P$ and $P^{\prime}$ at $\left(k_{x}, k_{y}\right)=$ $( \pm 0.5,0) \quad(\pi / T)$, where $A= \pm 0.5$. However, the parameter $B$ in equation (8) depends on $\omega$, e.g it is different for each value of $\omega$, indicating a change in the geometrical shape of the Fermi loops at every value of $\omega$. When $\omega=0.1$, and $B=0.45$ (it is measured in units $\left(\frac{\pi}{T}\right)^{2} \cdot v_{F}^{2}$ ). $B$ is here smaller than the value of the parameter $A$, then the Fermi surface consists of two loops (see Fig. 3). As $\omega$ increases, the value of the parameter $B$ also increases, i.e. to $B=0.48 \approx A$ at $\omega=0.3$. Then the Fermi surface has the shape of Lemniscate of Bernoulli (see Fig. 3). When the value $\omega=0.5$, the value $B=0.8$, which is now larger than the value of $A$. Here the Fermi surface consists of one loop having a shape of Cassini oval (see Fig. 3, middle). At the next increasing value of $\omega>0.5$, another Lifthitz quantum transition occurs, where the Fermi surface is transformed from the Cassini oval into three Fermi loops (see Fig. 3).

At intermediate frequencies. When $\omega$ increases and it is higher than 0.5 the two circles associated with Dirac cones first slightly deformed towards each other and then overlap and transformed into a new single elongated oval. With further increasing frequency $\omega$ this elongated Fermi surface evolves from a single quadratic Cassini oval to a pair of ovals. In this frequency range when $\omega \neq 1$ a pair of 2D Weyl points, associated with two ovals located on the sides shown in Figure 3 do arise. Meanwhile in a middle between these two ovals there arises a very elongated Fermi surface in the form of Cassini oval. Therewith, it is constituting the Lifshitz quantum phase transition. As the frequency increases further, these pair of side ovals change slightly their positions as shown in Figure 4. We associate that structure with the Dirac monopole or antimonopole which are connected by a Dirac string. That interaction between the monopole and the anti-monopole affects the Dirac cones anisotropy.

At the value $\omega=1$, the middle oval shows a special case where the spectrum has three zero energy points. The more detail consideration reveals that in the vicinity of the middle zero-energy point, that is at $K$ point of BZ, i.e. $\left(\varepsilon=0\right.$ at $\left.k_{x}=k_{y}=0\right)$ the spectrum shows a 


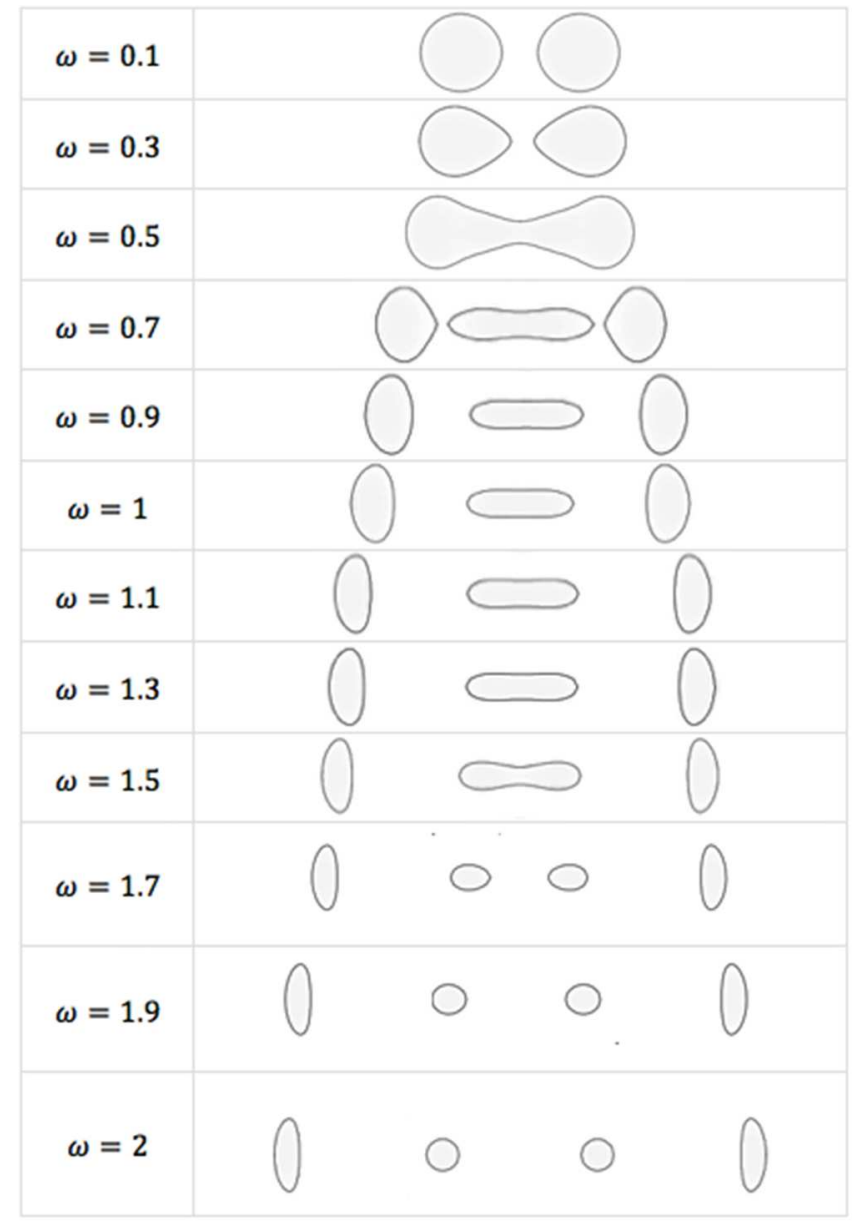

Fig. 3. The evolution of Fermi surface for graphene inside linear resonator, when its resonating frequency, $\omega$, increases. The Fermi surface is created by a cross-section of Dirac cones by a plane of the constant quasi-energy. $\omega$ is expressed in the units of $\pi / T$. The Fermi surface has a form of loops and Cassini ovals. The electric and magnetic fields in the resonator can be created by the application of static magnetic field and by two beams of the linear polarised laser light, which have oppositely oriented momenta. In the calculations we have used the following parameters: $\theta=0, A_{0}=1, \mu_{H}=1$ and $A_{0}^{H}=1$. Here, with each oval a $2 \mathrm{D}$ Weyl point is associated. Note that with rising frequency value there new Weyl points are created, and always in pairs, having opposite circulation. Depending on the sign of this circulation (positive or negative), the Weyl point is the Dirac monopole or anti-monopole. At intermediate frequencies, $0.5<\omega<1.7$, we see, that the new two "baby" Weyl points just about to be created and they are connected by Dirac string (presented by an elongated middle oval). At the critical value of $\omega$ these new Weyl points appears, there the strong(infinite) anisotropy of the associated Dirac cones arises.

parabolic dependence on the momentum $k_{y}$ (see Fig. 5a) and a linear dependence on $k_{x}$ (Fig. 5b). This fact can be interpreted as divergence of anisotropy $\alpha$ at the zeroenergy point, which is here arises at $K$ or $K^{\prime}$ points of BZ. These parabolic and linear dependencies along orthogonal axis in the momentum space indicate effectively an

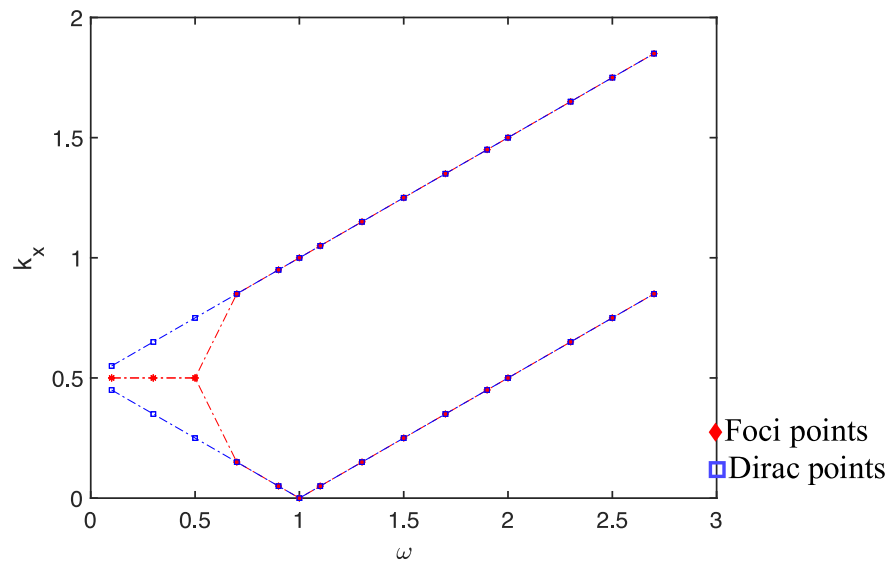

Fig. 4. The positions of Cassini ovals foci points as well as Dirac points in the quasi-energy, momentum space. Here only the positive momenta, $k_{x}>0$, are presented. In these calculations the values of the parameters were taken as $\theta=0$, $A_{0}=1, \mu_{H}=1$ and $A_{0}^{H}=1$.

appearance of strong anisotropy. It is formed by an odd number of zero energy points: by two Weyl points and one having highly anisotropic spectrum arising due to the linear and parabolic contributions.

The more detail investigation of the ovals just in the close vicinity of the Dirac points placed on the sides show that there has the form of ellipses for all $\omega$. Figure 6 presents this elliptical shape at $\omega=1$ (here the constant energy plane is cutting the cone perpendicular to its axis). The ellipse has a central point, $\left(k_{x}, k_{y}\right)=(1,0)$, which is the zero energy point at this case, and the curve can be described by $\frac{k_{x}^{2}}{b}+\frac{k_{y}^{2}}{a}=1$ with anisotropy $\alpha=\frac{a}{b}=2.1$. (The value $\alpha$ represents the aspect ratio of geometrical shapes, where $\alpha=1$ is a circle, $1<\alpha \leq 2$ is an ellipse, and $\alpha>2$ is a super-ellipse, etc.) As the frequency increases, the Dirac cones deformed more, hence the aspect ratio increases (see Fig. 7).

The similar transformations of the quasi-energy spectrum and Fermi surface for a slightly doped graphene arise in this resonator when the intensity of the electromagnetic field or its amplitude, $A_{0}$, increases. The effect of the Fermi surface splitting is also leading to a series of the Lifshitz quantum phase transition. These phenomena have been here investigated for a range of the field amplitudes $\left(A_{0}\right)$ and, indeed, show the strong similarity with effects described in the previous section, when the frequency $\omega$ changes. Besides, the increasing intensity of the field in the resonator results in a direct opening and closing of gap in the centre of the spectrum (see Fig. 8). To illustrate the effect, we start from the special case, when $A_{0}=1$ (in units of $\frac{\pi}{T} \cdot v_{F}$ ) and $\omega=1$. The spectrum here is presented in Figure 8. For amplitudes $A_{0}<1$, the middle point is transformed to a non-zero energy (see Fig. 8a), producing a gap, which is inversely proportional to $A_{0}$. As the amplitude increases further the gap becomes smaller and smaller it reaches zero at $A_{0}=1$. When $A_{0}<1$ the spectrum has two Dirac cones/points. The value $A_{0}=1$ is 


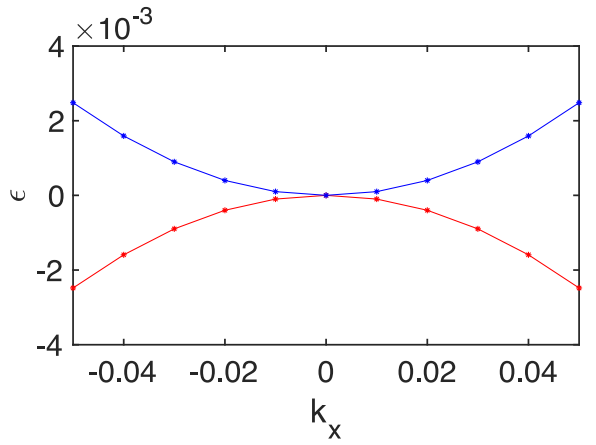

(a)

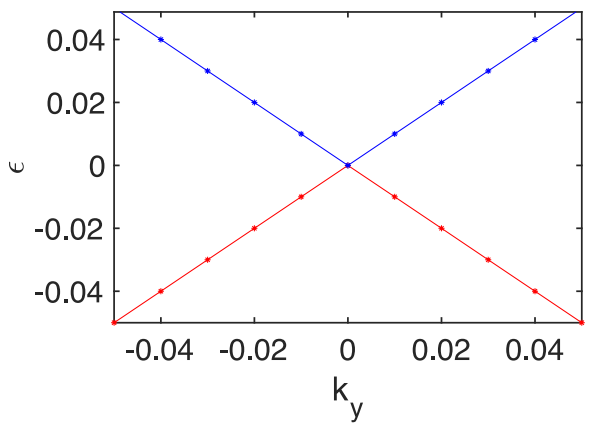

(b)

Fig. 5. (a) A cross-section of quasi-energy spectrum in the plane $k_{y}=0$, i.e. the dependence, $\varepsilon\left(k_{x}, 0\right)$. It shows parabolic behaviour: $\varepsilon \propto k_{x}^{2}$. (b) A cross-section of quasi-energy spectrum in the plane $k_{x}=0$, i.e. the dependence, $\varepsilon\left(0, k_{y}\right)$. It shows lineartype dependence $\varepsilon \propto\left|k_{y}\right|$. These qualitative differences in the energy momentum behaviour indicate on an infinite anisotropy arising in the vicinity of the point: $k_{x}=k_{y}=0$.

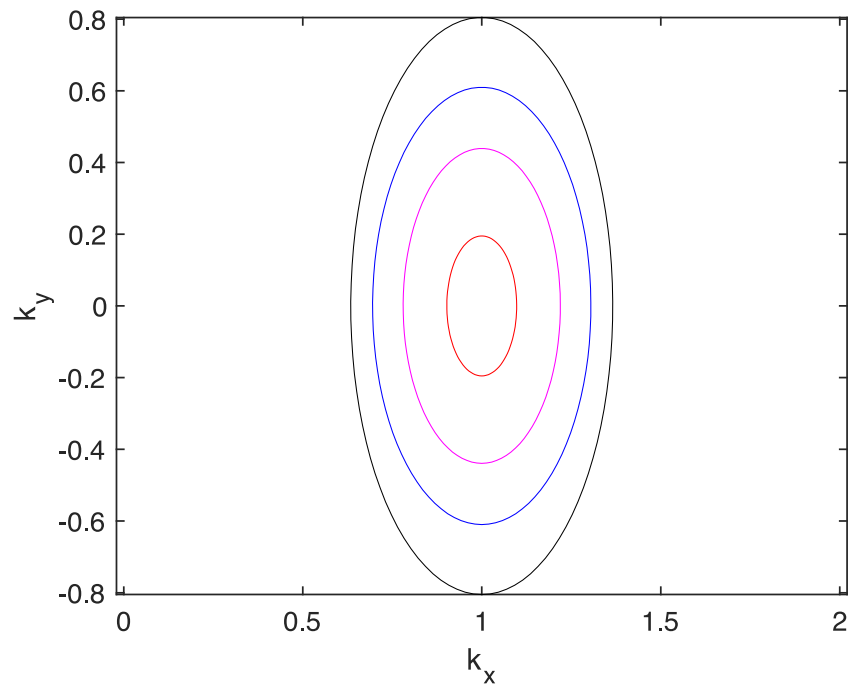

Fig. 6. The cross-section of the energy spectrum in for graphene in linear resonator. It is presented for the Dirac cone located on a side of the spectrum. There is static magnetic field and linear polarised dynamic fields (standing wave) with the frequency $\omega=1$ inside resonator. The ellipses have a fixed anisotropy of $\alpha=2.1$, that represents a deformation of the Dirac cone.

critical. There, besides two existing Dirac points emerges the third zero energy point. When $A_{0}>1$ this point is splitting into two 2D Weyl points with opposite circulations. As a result, we have here already four Weyl points. These points move apart from the centre as the amplitude $A_{0}$ increases (see Fig. 8b). With each of these zero energy points of spectrum there are associated anisotropic Dirac cones, which change shape and position as $A_{0}$ varies. The spectrum cross sections at a fixed energy which is related to the Fermi energy for the slightly doped graphene has a form of Cassini ovals. With its transformations there arise a series of Lifshitz quantum phase transitions which starts with the phase where the Fermi energy consists of one pair

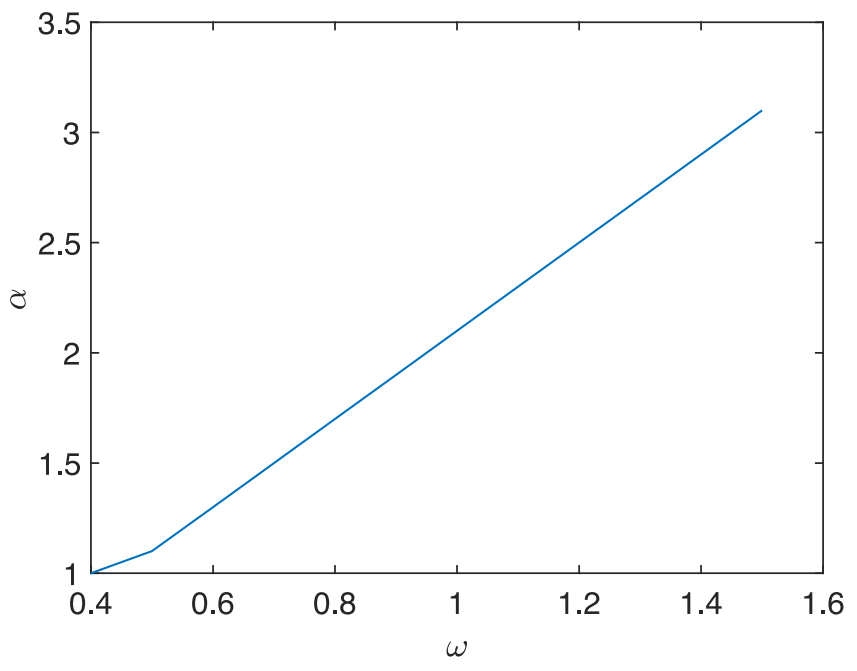

Fig. 7. The aspect ratio of the cross section or the anisotropy parameter for a (side) Dirac cone as a function of electromagnetic laser field frequency $\omega$ in the units of $\pi / T$. The parameters used in the calculations equal to $\theta=0, A_{0}=1$, $\mu_{H}=1$ and $A_{0}^{H}=1$.

of ovals. Then with increasing intensity the Lifshitz quantum phase transition arise at which the form of Fermi surface transforms into the two pairs of Cassini ovals (see Fig. 9). For stronger intensity, at the critical $A_{0}=1.9$ another such quantum transition occurs. There the middle pair of the Fermi surface loops splits and merges with the side pair (see Fig. 9). The change in the positions of Dirac pairs is presented in Figure 10. As we see from this figure their number increases for amplitudes larger than $A_{0}=1$. The cross-sections of the constant energy for side cones form ellipses with aspect ratio or the anisotropy that decreases as the amplitude $A_{0}$ increases (see Fig. 11). Similar transformations of quasi-energy spectrum happen when instead of static periodic magnetic field we apply static periodic electrical field, that is when $U_{0}>0$. 


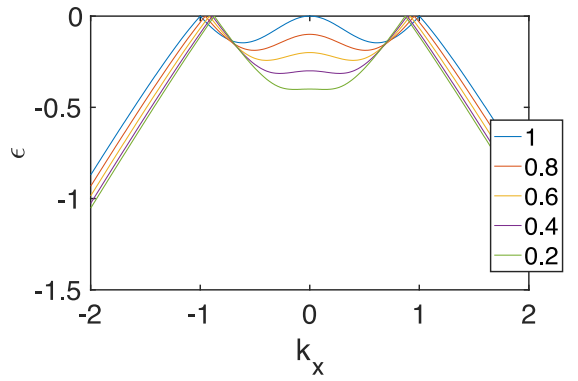

(a)

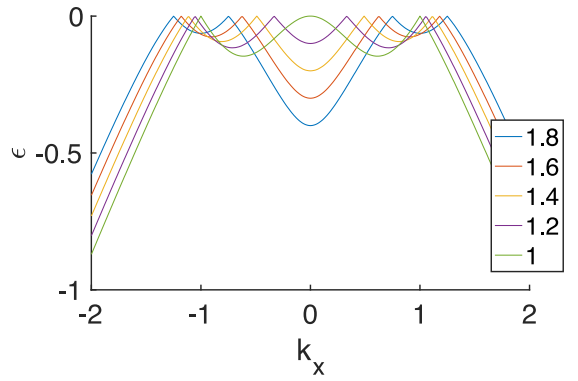

(b)

Fig. 8. The graphene inside linear resonator subjected to a static magnetic field and a linearly polarised electromagnetic field from laser forming a standing wave inside the resonator. The cross-sections of the quasi-energy spectrum $\varepsilon\left(k_{x}, k_{y}\right)$ by the plane $k_{y}=0$ taken at different value of the amplitude $A_{0}=0.2 ; 0.4 ; 0.6 ; 0.8 ; 1.0 ; 1.2 ; 1.4 ; 1.6 ;$ and 1.8 . It is measured in the units of $\pi / T$. The spectrum was calculated when the parameters $\theta=0, \omega=1, \mu_{H}=1$ and $A_{0}^{H}=1$. (a) Shows the modification of the spectrum where at zero $k_{x}$ momentum and at the value $A_{0}=1.0$ there arises the parabolic zero energy point; (b) illustrates a splitting of the parabolic zero energy point at $k_{x}=0$ (in the middle) into two Weyl points (sharp corners on the Figure) which are moving apart when the amplitude of the electromagnetic field in the resonator, $A_{0}$ increases further $\left(A_{0}>1\right)$. At the same time, the side Weyl points are shifting toward the new ones.

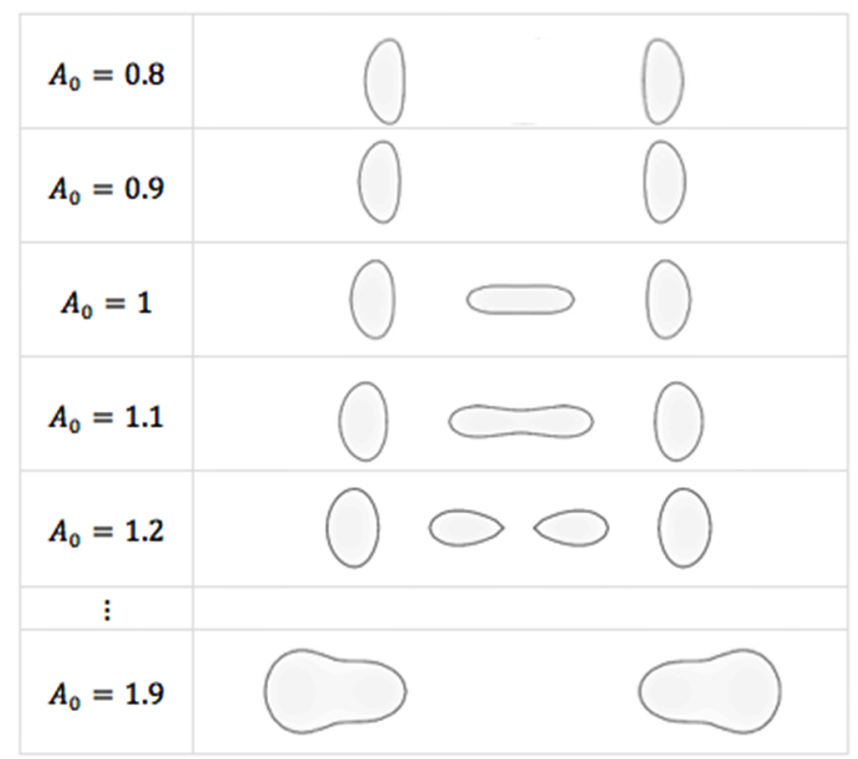

Fig. 9. The evolution of Fermi surface for graphene inside linear resonator, when the amplitude of the electromagnetic field, $A_{0}$, increases. That evolution indicates that there arise three Lifshitz quantum phase transitions. For the first phase of small amplitudes $A_{0}<1$, the Fermi surface consists of two Cassini ovals. Then, at $A_{0}=1$, the Lifshitz quantum phase transition occurs and there in the Fermi surface appears additional very elongated loop. In the range of $1.1<A_{0}<1.2$, these loops split into two and for the next phase the Fermi surface consists of 4 elliptical-like loops. Finally, at large amplitudes $1.2<A_{0}$ in another quantum phase transition the Fermi surface again is transformed into two loops. $A_{0}$ is here measured in the units of $\pi / T$. In these calculations we used the following parameters $\theta=0, \omega=1, \mu_{H}=1$ and $A_{0}^{H}=1$.

\section{Conclusion}

In this paper, we highlighted the relativistic properties of graphene by changing the Dirac-Cone spectrum of charge

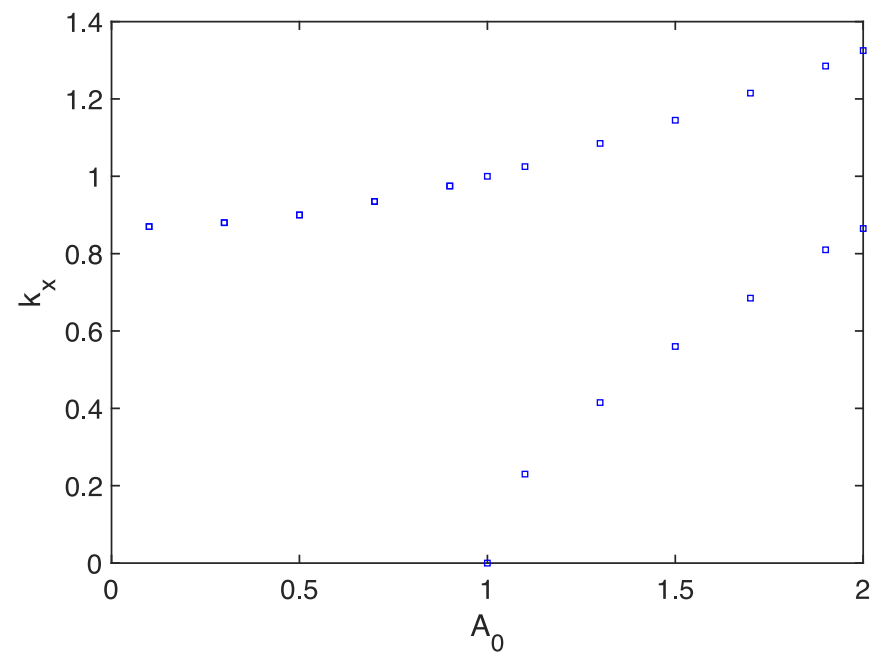

Fig. 10. The positions of Dirac points in the Brillouin zone for graphene driven by both the linear polarised laser light and static magnetic field as the amplitude $A_{0}$ changes. $\theta=0$, $\omega=1, \mu_{H}=1$ and $A_{0}^{H}=1$.

carriers. The theoretical modification of the spectrum is approached by applying high-frequency laser fields in the presence of static electric/magnetic fields. The number of Dirac points, the shape of Dirac cones and the gap in spectrum can all be controlled by changing the laser's parameters. In different potentials the spectrum shows different anisotropy, which can be further changed in specific potentials applications by changing their parameters. Thus, the modification of the anisotropy of the Dirac cone causes destruction/creation of Dirac points, hence optoelectronic graphene properties can be modified. We have demonstrated that anisotropic structures in monolayer graphene can be controlled by changing high-frequency lasers' parameters, which even allow generation of infinite anisotropy. Moreover, we have also shown that for the slightly doped graphene there may arise Lifshitz 


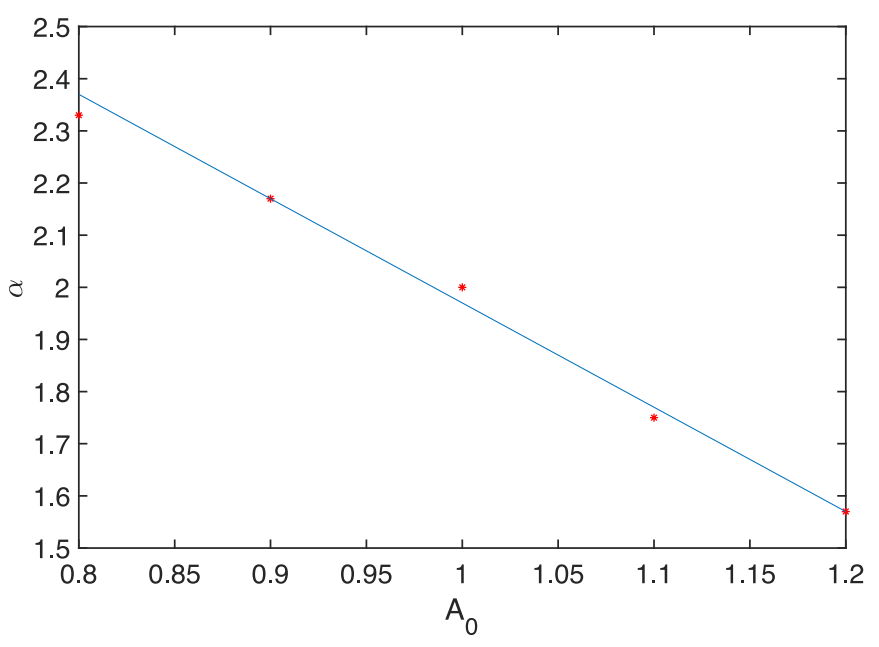

Fig. 11. The dependence of aspect ratio of the Dirac side cones anisotropy $\alpha$ on the laser's light amplitude $A_{0}$ measured the units of $\pi / T$. Here $\theta=0, \omega=1, \mu_{H}=1$ and $A_{0}^{H}=1$.

quantum phase transitions where the shape of the Fermi surface takes dramatic topological transformations when the amplitude or the frequency of the laser light changes. In recent experiments, graphene layer formed on $\mathrm{SiC}$ has been irradiated with laser light of the different frequencies [23]. The paper reported a discovery of remarkable photo-physical phenomena arising in graphene with the laser irradiation. There it was found that the electrical resistance of graphene increases under light illumination in contrast to conventional materials where it normally decreases. One simple explanation may come from the results of the present paper, namely, by an opening of the gap in the Dirac spectrum, the resistance of the graphene must increase. An interesting question arises how this gap will be closed when the laser irradiation is stopped. We expect that the future studies will address this issue.

The work of F.V.K. was supported by the Government of the Russian Federation through the ITMO Professorship Program and Royal Society Grant: Growth of GeSiSn/Si Multilayers, Quantum Dots and Topological Insulators.

\section{Author contribution statement}

Shahd Alfadhli calculated all spectra and wrote the draft of the manuscript. Sergey Saveliev and Feo Kusmartsev analysed data. Feo Kusmartsev significantly rewrote the final version of the manuscript.
Open Access This is an open access article distributed under the terms of the Creative Commons Attribution License (http://creativecommons.org/licenses/by/4.0), which permits unrestricted use, distribution, and reproduction in any medium, provided the original work is properly cited.

\section{References}

1. K.S. Novoselov et al., Science 306, 666 (2004)

2. J.C. Meyer, A.K. Geim, M.I. Katsnelson, K.S. Novoselov, T.J. Booth, S. Roth, Nature 446, 60 (2007)

3. A. O'Hare, F.V. Kusmartsev, K.I. Kugel, Nano Lett. 12, $1045(2012)$

4. A.C. Neto, F. Guinea, N.M. Peres, K.S. Novoselov, A.K. Geim, Rev. Mod. Phys. 81, 109 (2009)

5. F.V. Kusmartsev, A.M. Tsvelik, JETP Lett. 42, 257 (1985)

6. S.E. Savel'ev, A.S. Alexandrov, Phys. Rev. B 84, 035428 (2011)

7. P. Rodriguez-Lopez, J.J. Betouras, S.E. Savel'ev, Phys. Rev. B 89, 155132 (2014)

8. R.D.Y. Hills, A. Kusmartseva, F.V. Kusmartsev, Phys. Rev. B 95, 214103 (2017)

9. Y.P. Bliokh, V. Freilikher, S. Savel'ev, F. Nori, Phys. Rev. B 79, 075123 (2009)

10. T.G. Pedersen, C. Flindt, J. Pedersen, N.A. Mortensen, A.P. Jauho, K. Pedersen, Phys. Rev. Lett. 100, 136804 (2008)

11. J.C.W. Song, A.V. Shytov, L.S. Levitov, Phys. Rev. Lett. 111, 266801 (2013)

12. K.N. Alekseev, E.H. Cannon, J.C. McKinney, F.V. Kusmartsev, D.K. Campbell, Physica D 113, 129 (2001)

13. H.T. Grahn, Semiconductor superlattices: growth and electronic properties (World Scientific, 1995)

14. J. Cayssol, C. R. Phys. 14, 760 (2013)

15. M. Engel et al., Nat. Commun. 3, 906 (2012)

16. Y. Fan, F. Zhang, Q. Zhao, Z. Wei, H. Li, Opt. Lett. 39, $6269(2014)$

17. S.M. Rao, J.J. Heitz, T. Roger, N. Westerberg, D. Faccio, Opt. Lett. 39, 5345 (2014)

18. X. Gan, R.J. Shiue, Y. Gao, S. Assefa, J. Hone, D. Englund, IEEE J. Sel. Top. Quant. Electr. 20, 95 (2014)

19. T. Wenger, G. Viola, J. Kinaret, M. Fogelström, P. Tassin, 2D Mater. 4, 025103 (2017)

20. S.E. Savel'ev, W. Häusler, P. Hänggi, Phys. Rev. Lett. 109, 226602 (2012)

21. S.A. Alfadhli, S.E. Savel'ev, F.V. Kusmartsev, J. Phys.: Conf. Ser. 961, 012012 (2018)

22. B. Dietz et al., Phys. Rev. B 88, 104101 (2013)

23. A.B.G. Trabelsi, F.V. Kusmartsev, M.B. Gaifullin, D.M. Forrester, A. Kusmartseva, Nanoscale 9, 11463 (2017) 